\title{
ON A RAMANUJAN'S IDENTITY
}

\author{
by MANVENDRA TAMBA
}

(Received 29 June, 1990)

1. Introduction. In [4], Ramanujan stated the following beautiful identity which was later proved by Bailey [2] and [3];

$$
q \prod_{n=1}^{\infty} \frac{\left(1-q^{5 n}\right)^{5}}{\left(1-q^{n}\right)}=\sum_{n=0}^{\infty} \frac{q^{5 n+1}}{\left(1-q^{5 n+1}\right)^{2}}-\frac{q^{5 n+2}}{\left(1-q^{5 n+2}\right)^{2}}-\frac{q^{5 n+3}}{\left(1-q^{5 n+3}\right)^{2}}+\frac{q^{5 n+4}}{\left(1-q^{5 n+4}\right)^{2}} .
$$

We denote by $S(n)$ the coefficient of $q^{n}$ on the right hand side of (1). The object of this paper is to prove the following two theorems.

THEOREM 1. (a) $S(n)$ is multiplicative. (b) Let $p$ be a prime. We have the following:

(i) if $p=5$, then $S\left(5^{n}\right)=5^{n}$;

(ii) if $p \equiv \pm 1(\bmod 5)$, then $S\left(p^{n}\right)=\left(p^{n+1}-1\right) /(p-1)$;

(iii) if $p \equiv \pm 2(\bmod 5)$, then $S\left(p^{n}\right)= \begin{cases}\left(p^{n+1}-1\right) /(p+1) & \text { if } n \text { is odd } \\ \left(p^{n+1}+1\right) /(p+1) & \text { if } n \text { is even. }\end{cases}$

THEOREM 2. Let $R(n)$ denote the number of integral solutions of the Diophantine equation

$$
5\left(x_{1}^{2}+x_{2}^{2}+x_{3}^{2}+x_{4}^{2}-x_{1} x_{2}-x_{2} x_{3}-x_{3} x_{4}\right)+x_{1}+x_{2}+x_{3}+x_{4}=n .
$$

Then

$$
R(n)=S(n+1)
$$

2. Proof of Theorem 1. For $1 \leq k \leq 4$, define

$$
\sigma_{k}(n)=\sum_{\substack{d \mid n \\ d=k(\bmod 5)}} n / d
$$

and note that

$$
\sum_{n=0}^{\infty} \frac{q^{5 n+k}}{\left(1-q^{5 n+k}\right)^{2}}=\sum_{n=1}^{\infty} \sigma_{k}(n) q^{n}
$$

As $S(n)$ denotes the coefficients of $q^{n}$ on the right hand side of (1), we have

$$
S(n)=\sigma_{1}(n)-\sigma_{2}(n)-\sigma_{3}(n)+\sigma_{4}(n) .
$$

Now, the part (a) of the Theorem 1 easily follows by observing that

$$
S(n)=\sum_{d \mid n}(n / d) \chi(d)
$$

where $\chi$ is the Dirichlet character defined by $\chi(2)=-1$. Part (b) of the Theorem 1 follows by using (2). 
3. Proof of Theorem 2. In this section we shall prove the following lemma from which Theorem 2 follows immediately.

Lemma. For $|q|<1$,

$$
\prod_{n=1}^{\infty} \frac{\left(1-q^{5 n}\right)^{5}}{\left(1-q^{n}\right)}=\sum_{n=0}^{\infty} R(n) q^{n}
$$

Proof. First we note that

$$
\sum_{n \in \mathbb{Z}} z^{A n} q^{B n^{2}+C n}=\prod_{n=1}^{\infty}\left(1-q^{2 B n}\right)\left(1+z^{A} q^{2 B n+C-B}\right)\left(1+z^{-A} q^{2 B n-C-B}\right),
$$

by Jacobi's triple product identity $[1$, p. 21$]$. Hence $\prod_{n=1}^{\infty}\left(1-q^{n}\right)^{-1}$ is the constant term (i.e. the term independent of $z$ ) in

$$
\prod_{n=1}^{\infty}\left(1+z q^{n-2}\right)\left(1+z^{-1} q^{n+1}\right)
$$

Now consider

$$
\begin{aligned}
& \sum_{y_{1}, y_{2}, y_{3}, y_{4}, y_{5} \in \mathbb{Z}}\left\{q^{5 / 2\left(\sum_{i=1}^{s} y_{i}^{2}\right)+\frac{1}{2}\left(y_{1}+y_{2}+3 y_{3}+5 y_{4}+7 y_{5}\right)} z^{y_{1}-y_{2}-y_{3}-y_{4}-y_{5}}\right\} \\
= & \left\{\prod_{n=1}^{\infty}\left(1-q^{5 n}\right)\left(1+z q^{5 n-2}\right)\left(1+z^{-1} q^{5 n-3}\right)\right\}\left\{\prod_{n=1}^{\infty}\left(1-q^{5 n}\right)\left(1+z^{-1} q^{5 n-2}\right)\left(1+z q^{5 n-3}\right)\right\} \\
& \times\left\{\prod_{n=1}^{\infty}\left(1-q^{5 n}\right)\left(1+z^{-1} q^{5 n-1}\right)\left(1+z q^{5 n-4}\right)\right\}\left\{\prod_{n=1}^{\infty}\left(1-q^{5 n}\right)\left(1+z^{-1} q^{5 n}\right)\left(1+z q^{5 n-5}\right)\right\} \\
& \times\left\{\prod_{n=1}^{\infty}\left(1-q^{5 n}\right)\left(1+z^{-1} q^{5 n+1}\right)\left(1+z q^{5 n-6}\right)\right\} \\
= & \prod_{n=1}^{\infty}\left(1-q^{5 n}\right)^{5}\left(1+z q^{n-2}\right)\left(1+z^{-1} q^{n+1}\right) .
\end{aligned}
$$

By comparing the constant terms on both sides of (3) we have $y_{1}=y_{2}+y_{3}+y_{4}+y_{5}$. Hence by making the change of variables

$$
x_{1}=y_{2}+y_{3}+y_{4}+y_{5}, \quad x_{2}=y_{3}+y_{4}+y_{5}, \quad x_{3}=y_{4}+y_{5}, \quad x_{4}=y_{5}
$$

to get the polynomial in the form given in Theorem 2 , the lemma follows.

ACKNOWLEDGEMENT. The author is very pleased to acknowledge the referee of this paper for his valuable suggestions.

\section{REFERENCES}

1. G. E. Andrews, The theory of partitions, Encyclopedia of Math. Appl., Vol. 2 (AddisonWesley, 1976).

2. W. N. Bailey, A note on two of Ramanujan's formulae, Quart. J. Math. 3 (1952), 29-31. 
3. W. N. Bailey, A further note on two of Ramanujan's formulae, Quart. J. Math. 3 (1952), $158-160$.

4. S. Ramanujan, Congruence properties of $p(n)$, (unpublished manuscript), Trinity College Library (1920).

Ramanujan Institute for Advanced Study in Mathematics, UNIVERSITY OF MADRAS,

MADRAS-600 005, INDIA 